\title{
VALORACIÓN DE DESECHOS DE BANANO (MASA ACUMINATA CAVENDISH SUBGROUP) Y CACAO (THEOBROMA CACAO) MEDIANTE PRODUCCIÓN DE COMPOST Y BIOL
}

EVALUATION OF BANANA WASTE (MASA ACUMINATA CAVENDISH SUBGROUP) AND COCOA (THEOBROMA CACAO), THROUGH THE PRODUCTION OF COMPOST AND BIOL

\begin{abstract}
Diego Muñoz Naranjo
Docente, Universidad Agraria del Ecuador, (Ecuador).

E-mail: dmunoz@uagraria.edu.ec ORCID: https:// orcid.org/0000-0003-2203-0588
\end{abstract}

Garolina Cuenca Poveda

Consultoría Ambiental (Ecuador).

E-mail: nicole6_cncp@hotmail.com ORCID: https://orcid.org/0000-0001-5401-0704

Garlos Banchón Bajaña

Docente, Universidad Agraria del Ecuador, (Ecuador).

E-mail: cbanchon@uagraria.edu.ec ORCID: http://orcid.org/0000-0002-0388-1988

Gustavo Pazos Galeas

Docente, Universidad de Guayaquil, (Ecuador).

E-mail: mdbpazos.cs5@gamil.com ORCID: https://orcid.org/0000-0001-7360-0937

Gitación sugerida:

Muñoz, D., Guenca, C., Banchón, G., y Pazos, G. (2020). Valoración de desechos de banano (Masa Acuminata Cavendish Subgroup) y cacao (Theobroma cacao) mediante producción de compost y biol. 3C Tecnología. Glosas de innovación aplicadas a la pyme, 9(1), 17-29. http://doi.org/10.17993/3ctecno/2020.v9n1 e33.17-29 


\section{RESUMEN}

El presente proyecto se centra en el aprovechamiento ecológico de desechos de banano (Musa Acuminata Cavendish Subgroup) y cacao (Theobroma cacao), tomando como caso de estudio la hacienda Secadal del cantón Naranjal (Guayas-Ecuador). En apenas cinco semanas, la mezcla de B. subtillis, melaza, Trichoderma, desechos de cacao y banano, y tierra para sembrar, fue el mejor tratamiento para mineralizar la materia orgánica y así producir una fracción sólida considerada como compost y una fracción líquida considerada como biol. Se reportan los siguientes resultados: $\mathrm{pH}$ cercano a 7, temperatura mesofilica, contenidos de materia orgánica $>7,6 \%$, carbono $>4,40 \%$, conductividad eléctrica $>11,38 \mathrm{mS} / \mathrm{cm}$, sólidos totales $>67,89 \%$ y sólidos volátiles $>25,34 \%$. De la cantidad de desechos orgánicos tratados, aproximadamente un $50 \%$ fue compost y $22 \%$ biol.

\section{PALABRAS CLAVES}

Biol, Desechos orgánicos, Biotransformación, Eco-balance, Digestión anaeróbica.

\section{ABSTRACT}

The present project focuses on the ecological use of banana waste (Musa acuminata Cavendish Subgroup) and cocoa (Theobroma cacao), taking as a case study the Secadal farm of the Naranjal canton (Guayas-Ecuador). In just five weeks, the mixture of B. subtillis, molasses, Trichoderma, cocoa and banana wastes, and land for sowing, was the best treatment to mineralize organic matter and thus produce a solid fraction considered as compost and a liquid fraction considered as biol. The following results are reported: $p H$ close to 7 , mesophilic temperature, organic matter contents $>7.6 \%$, carbon $>$ $4.40 \%$, electrical conductivity $>11.38 \mathrm{mS} / \mathrm{cm}$, total solids $>67.89 \%$ and solids volatile $>25.34 \%$. Of the amount of organic waste treated, approximately $50 \%$ was compost and $22 \%$ biol.

\section{KEYWORDS}

Biol, Organic waste, Biotransformation, Eco-balance, Anaerobic digestion. 


\section{INTRODUCCIÓN}

En las últimas décadas, la población mundial aumentó a 6.000 millones de habitantes, trayendo como consecuencia que los países de altos ingresos generen alrededor de 683 millones de toneladas de desechos, y esto solo representa un $34 \%$ de desechos a nivel mundial (Aslam et al., 2019; Kaza et al., 2018). Se han proyectado 3,40 billones de toneladas de desechos para 2050, en donde los desechos generados por persona promediarían a 4,54 Kg diarios (Aslam et al., 2019; FAO, 2019). Contribuyen también las malas prácticas agrícolas, puesto que alrededor del $14 \%$ de los alimentos producidos se pierde desde la postcosecha hasta la etapa de comercio minorista (FAO, 2019; Ferronato y Torretta, 2019; Paritosh et al., 2017). Por ejemplo, el cacao es uno de los primeros eslabones de la cadena agroindustrial mundial generando más de 120 mil millones de dólares, pero también con grandes aportes al desecho agrícola (Arvelo et al., 2016).

La gestión de residuos sólidos (GRS) en los países en desarrollo representa una verdadera preocupación ambiental y social pues la opción de "tratamiento" es solamente una disposición final en vertederos abiertos o en rellenos sanitarios inadecuados (Bayard et al., 2018; Ferronato et al., 2019) leaching behavior, contents in biochemical constituents (determined by Van Soest's acid detergent fiber method. Por ejemplo, en Ecuador, cada habitante produce en promedio alrededor de 0,58 kilogramos de residuos sólidos, de los cuales el 54\% es residuo orgánico (Morán, 2018). En específico, tomando como ejemplo al cacao y al banano, principales motores de la economía de Ecuador, se ha tratado de buscar solución a la gestión de residuos agrícolas sin éxitos de gran impacto. Por tanto, en el presente trabajo se reporta la aplicación de medidas sostenibles basadas en el compostaje de desechos del banano y cacao de una pequeña hacienda de Ecuador.

\section{MATERIALES Y MÉTODOS}

Se recolectaron y secaron al sol desechos fibrosos del raquis de banano, cáscaras de cacao y cáscaras de naranja. Para el presente proyecto se utilizaron aproximadamente $2 \mathrm{Kg}$ de desechos orgánicos. Mediante 
trituración, se pulverizaron los desechos hasta un tamaño de partícula menor a $1 \mathrm{~cm}$. Los desechos fueron colocados en lechos de madera; cada lecho tuvo $37 \mathrm{~cm}$ de ancho y $23 \mathrm{~cm}$ de alto. Como cultivos iniciadores, se utilizaron disoluciones de Bacillus subtillis y Trichoderma (2 g del cultivo en un litro de agua). La mezcla de los triturados y cultivos iniciadores, así como desechos de cáscaras de naranja, y frutos en descomposición de banano, fueron mezclados y mezclados periódicamente, según los respectivos tratamientos que se muestran en la Tabla 1.

Tabla 1. Tabla de tratamientos.

\begin{tabular}{|c|c|c|c|c|}
\hline Componentes & Trat. 1 & Trat. 2 & Trat. 3 & Trat.4 \\
\hline (i) B. subtillis & $250 \mathrm{ml}$ & $250 \mathrm{ml}$ & - & - \\
\hline $\begin{array}{c}\text { (ii) Melaza } \\
\text { (iii) Trichoderma }\end{array}$ & $20 \mathrm{ml}$ & $20 \mathrm{ml}$ & $20 \mathrm{ml}$ & - \\
\hline $\begin{array}{c}\text { (iv) Desechos de cacao y } \\
\text { banano }\end{array}$ & $70 \mathrm{ml}$ & - & $70 \mathrm{ml}$ & - \\
\hline (v) Tierra para sembrar & $100 \mathrm{~g}$ & $100 \mathrm{~g}$ & $100 \mathrm{~g}$ & $100 \mathrm{~g}$ \\
\hline (vi) Banano (Fruto) & - & $370 \mathrm{~g}$ & - & $92,5 \mathrm{~g}$ \\
\hline (vii) Cáscara de naranja & - & - & $370 \mathrm{~g}$ & $92,5 \mathrm{~g}$ \\
\hline
\end{tabular}

El pH y la temperatura fueron medidos periódicamente en cada lecho mediante sondas paramétricas. La concentración de carbono y materia orgánica se determinó mediante titulación. Se agregó 0,5 g de muestra y $5 \mathrm{ml}$ de dicromato de potasio y ácido sulfúrico dejándolos por 30 minutos; luego, se agregó $35 \mathrm{ml}$ de agua destilada, y bajo calentamiento a $100^{\circ} \mathrm{C}$ se mantuvo durante 1 hora. Una vez que se terminó el proceso, esto fue filtrado y colocado en una celda, para medición en espectrofotómetro.

Para la determinación del porcentaje de sólidos totales (ST), se utilizó el método ISO11465:1993. Se pesó aproximadamente $20 \mathrm{~g}$ de muestra sólida en una cápsula de porcelana seca y pre-pesada en una balanza analítica (Sartorius, USA). Esta muestra se secó en una estufa (POL-EKO) a $105^{\circ} \mathrm{C}$ surante 24 horas. La muestra seca se enfrió en un desecador y se volvio a pesar. 
Para obtener el contenido de sólidos volátiles (SV) mediante gravimetría, se colocó la cápsula con muestra a $105^{\circ} \mathrm{C}$ en una mufla (Snol, USA). Se aumentó lentamente la temperatura hasta llegar a $550^{\circ} \mathrm{C}$, donde permaneció por 2 horas (calcinación). Luego se enfrió en un desecador para luego pesar la cápsula en una balanza analítica (Sartorius, USA).

Para establecer la normalidad de las poblaciones, se utilizó la prueba estadística de ANOVA. Se estableció el método de un solo factor dentro del programa MINITAB para así saber si existe una diferencia estadísticamente significativa entre las poblaciones. Cada una de estas pruebas se las evalúo con los resultados del valor de la probabilidad. Se utilizó el 95\% de nivel de confianza dentro de las pruebas. Debido a que son más de 2 poblaciones y el valor de $\mathrm{p}$ resulto $\mathrm{p}<0,010)$, también a la falta de muestras del proyecto, no se pudo completar el análisis propuesto: la prueba t-Student. Por ende, se realizó un análisis donde se establecieron los supuestos, lo que involucró a la normalidad en las poblaciones para establecer esta nueva estadística no paramétrica de Wilcoxon.

\section{RESULTADOS}

Se determinaron los cambios de $\mathrm{pH}$ y temperatura durante 5 semanas (Figuras 1 y 2). Todos los datos que se obtuvieron durante el muestreo fueron ingresados en el programa estadístico Minitab 16. Durante las 5 semanas, los lechos de compostaje liberaron calor, así como vapor de agua.

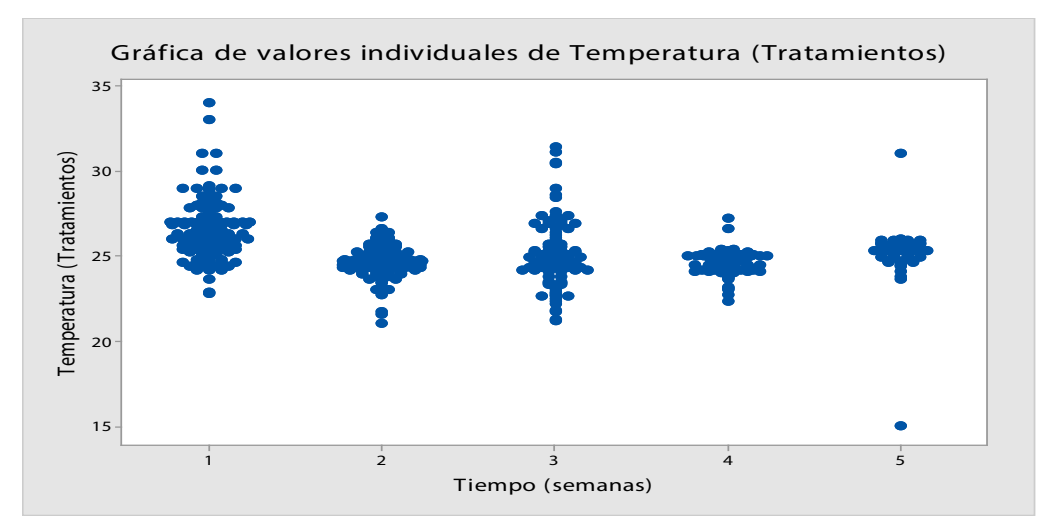

Figura 1. Cambio de temperatura en función de 5 semanas. Valores mostrados de todos los tratamientos. 


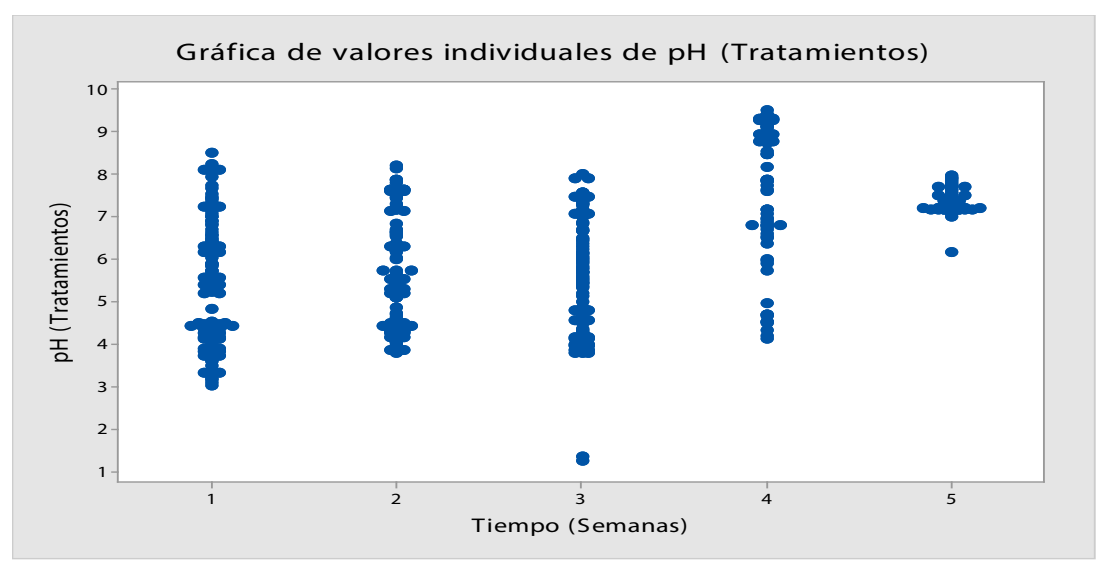

Figura 2. Cambio de pH para todos los tratamientos en 5 semanas.

Los tratamientos 1, 2 y 4, fueron los únicos que produjeron un producto sólido y uno líquido. En la Figura 3, del total de desechos orgánicos que fueron utilizados para el compostaje (1880 g), aproximadamente un 50,3\% (947,2 g) fueron materia sólida, considerada como compost según los resultados de materia orgánica y carbono total; además, de la cantidad total de muestra inicial, un 22,1% (415,3 g) estuvieron en estado líquido, lo que se consideraría como biol, según los análisis químicos realizados.

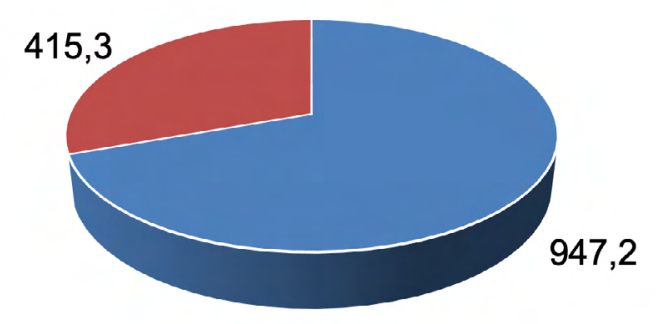

\section{- Compost - Biol}

Figura 3. Obtención de compost y biol a partir de desechos orgánicos. 
Como se observa en la Figura 4, el contenido de materia orgánica se mantuvo entre 8 y 14 \% en los cuatro tratamientos; y la cantidad de carbono en los tratamientos, estuvo entre 4 y $8 \%$. De los cuatro tratamientos, los tratamientos 1 y 2 obtuvieron mayores contenidos de materia orgánica y carbono, ambos con la adición de melaza, Trichoderma y Bacillus subtillis; se obtuvo una concentración de materia orgánica entre 8,86 y $11,25 \%$ y de carbono total entre 5,11 y $6,51 \%$; estos valores son primordiales debido a que éste establecerá la calidad del compost y el biol antes de que sean utilizados.

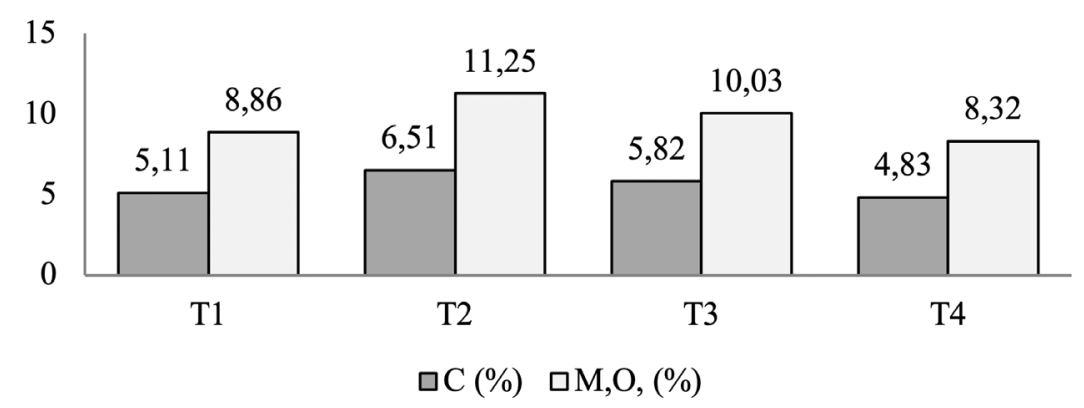

Figura 4. Porcentajes de análisis químico de carbono y materia orgánica.

Los tratamientos 1, 2 y 4, fueron los únicos que obtuvieron un producto líquido, conocido como biol. Como se observa, los valores más altos de conductividad eléctrica los obtuvo el tratamiento 2, con cantidades entre $45,9-52,6 \mathrm{mS} / \mathrm{cm}$.

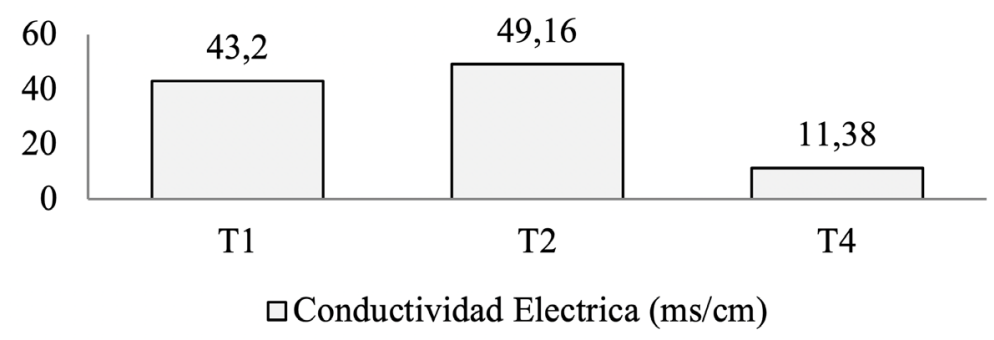

Figura 5. Tabla de conductividad eléctrica de los tratamientos T1, T2 y T4. 
En el presente estudio, se utilizó el método de calcinación para determinación de sólidos volátiles (SV), el cual consistió en llevar a la estufa a $105^{\circ} \mathrm{C}$ por un tiempo de 24 horas; luego después de este proceso se llevó a la mufla por un periodo de 2 horas, obteniendo resultados de estas pruebas un valor promedio de sólidos totales (ST) entre 67,89 - 81,28\%, que se encuentran presentes en la muestras de compost y biol, así mismo de SV entre 25,34 y 42,33\%.

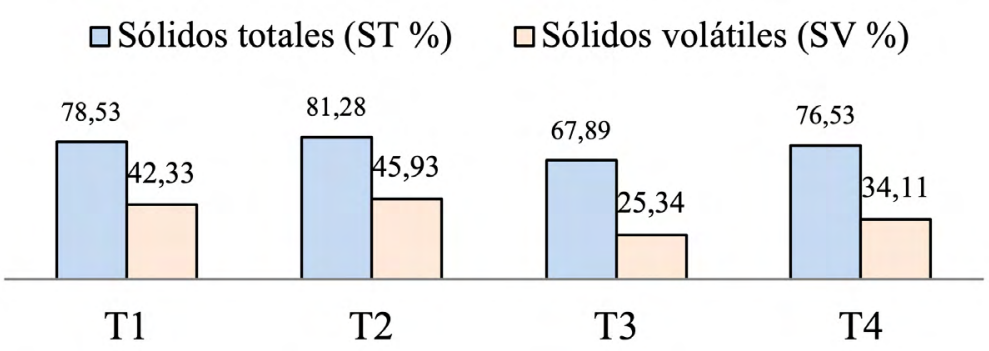

Figura 6. Porcentajes de sólidos totales y volátiles de los tratamientos.

\section{DISCUSIÓN}

La temperatura es el factor primordial para indicar el desarrollo del proceso de descomposición de la materia orgánica, pues este incremento en la temperatura facilita acelerar la descomposición y eliminar o reducir las poblaciones de los microorganismos existentes (Acosta et al., 2018; Arnawa et al., 2018; Chaniago y Ramlan, 2017; Fan et al., 2018). Como parámetro de control del proceso de compostaje, la temperatura debería oscilar entre $55-65^{\circ} \mathrm{C}$ cuando el proceso es termofílico. Según los resultados aquí presentados, el presente proceso está en el rango mesófilo (Ogunlade et al., 2019; Rahim et al., 2018; Vitinaqailevu y Rajashekhar Rao, 2019).

En cuanto al pH, éste varía según la composición microbiana y según los sustratos del lecho. Según los estándares de compostaje, el rango de pH establecido es 6,5 a 8,0 como evidencia de procesos de desmineralización de la materia orgánica volátil (Ogunlade et al., 2019). La acción de hongos y bacterias ayuda a la conformación de ácidos orgánicos que posteriormente reducen el pH (Arnawa et al., 2018). 
En el tratamiento 2 y 3, con melaza y Trichoderma, se obtuvo un $\mathrm{pH}$ menor a 4,84, mientras que los tratamientos con Bacillus subtillis ( 1 y 4 ) el pH se mantuvo entre 6,5 hasta 7,0, siendo estos tratamientos los aptos para utilización como producción de compost y biol.

Tabla 2. Valores de prueba para uso de compost y biol.

\begin{tabular}{|c|c|}
\hline \multicolumn{2}{|c|}{ CALIDAD DE COMPOSTAJE } \\
\hline Materia orgánica & $>30 \%$ \\
\hline Temperatura & $45-600 \mathrm{C}$ \\
$\mathrm{pH}$ & $6,5-8,5$ \\
Carbono & $15 \%$ \\
\hline
\end{tabular}

Fuente: Environmental Protection Agency (EPA).

La conductividad eléctrica corresponde al contenido de electrolitos que se encuentran presentes en el suelo y son solubles en el agua; la conductividad sirve también como un indicador de la salinidad del suelo (Chaniago y Ramlan, 2017). Aproximadamente entre 8 - 16 mS/cm se establece como fuertemente salino y mayor a $16 \mathrm{mS} / \mathrm{cm}$ como muy fuertemente salino (Dadi et al., 2019). Según los presentes resultados, los Tratamientos 1 y 2 fueron muy fuertemente salinos, a diferencia del Tratamiento 4.

Los sólidos volátiles (SV) garantizan la energía suficiente para elevar la temperatura en los procesos celulares. Si dividimos el contenido de sólidos volátiles en función de los sólidos totales (ST), obtendremos una relación que indicará la fracción de materia orgánica mineralizada. Según estándares internaciones de la Agencia de Protección Ambiental (EPA US), la producción de biosólidos se confirmaría cuando la relación entre SV y ST es mayor que un 0,60. Según los resultados experimentales, el Tratamiento 1 (Mezcla de B. subtillis, melaza, Trichoderma, desechos de cacao y banano, tierra para sembrar) obtuvo un 0,54 de relación SV/ST, muy cercana a lo establecido por la EPA. Tomando en cuenta que su pH estuvo dentro de los rangos recomendados, es esta mezcla la recomendada para la producción de compost y biol. Los otros tratamientos tienen la desventaja de ser muy ácidos y de mineralizar cantidades insuficientes de materia orgánica. 


\section{CONCLUSIONES}

En apenas cinco semanas, la mezcla de B. subtillis, melaza, Trichoderma, desechos de cacao y banano, y tierra para sembrar, fue el mejor tratamiento para mineralizar la materia orgánica y así producir una fracción sólida considerada como compost y una fracción líquida considerada como biol; esto a un rango mesófilo de temperatura. Los cambios de $\mathrm{pH}$ demuestran que no todos los procesos aquí estudiados permitieron una estabilización a un rango neutro de $\mathrm{pH}$. 7,0. Como perspectiva, el aprovechamiento integral de desechos agrícolas es de amplio potencial para su aplicación práctica, y así contribuir con la solución ambiental a problemáticas antiguas en tiempos modernos.

\section{REFERENCIAS BIBLIOGRÁFICAS}

Acosta, N., De Vrieze, J., Sandoval, V., Sinche, D., Wierinck, I., y Rabaey, K. (2018). Cocoa residues as viable biomass for renewable energy production through anaerobic digestion. Bioresource Technology, 265, 568-572. https://doi.org/10.1016/j.biortech.2018.05.100

Arnawa, I. K., Udiyana, B. P., Widnyana, I. K., y Sukerta, D. I. M. (2018). Utilization of Fermentation Technology for Processing Waste to be Composted and Biourine. International fournal of Contemporary Research and Review, 9(02). https://doi.org/10.15520/ijcrr/2018/9/02/420

Arvelo, M. Á., Delgado, T., Maroto, S., Rivera, J., Higuera, I., y Navarro, A. (2016). Estado actual sobre la producción y el comercio del cacao en América (N. ${ }^{\circ}$ 9292486179). Instituto Interamericano de Cooperación para la Agricultura (IICA), Centro de Investigación y Asistencia en Tecnología y Diseño del Estado de Jalisco A.C. (CATIEJ).

Bayard, R., Benbelkacem, H., Gourdon, R., y Buffière, P. (2018). Characterization of selected municipal solid waste components to estimate their biodegradability. Fournal of Environmental Management, 216, 4-12. https://doi.org/10.1016/j.jenvman.2017.04.087 
Aslam, H. M. U., Butt, M. A., Bareen, F., Shafiq, M., Butt, F. A., Shahid, M., Javaid, A., y Husnain, A. (2019). Organic Waste Composting: A Resource Recovery Approach towards Sustainable Environment. Proceedings of the World Conference on Waste Management, 1(2), 30-41. https:/ / doi.org/10.17501/26510251.2019.1203

Chaniago, R., y Ramlan, W. (2017). Utilizing Cocoa Rind as Organic Fertilizer to Support Sustainable Agriculture. Fournal of Tropical Soils, 21(1), 33-39. https://www.researchgate. net/publication/326739665_Utilizing_Cocoa_Rind_as_Organic_Fertilizer_to_Support_ Sustainable_Agriculture

Dadi, D., Daba, G., Beyene, A., Luis, P., y Van der Bruggen, B. (2019). Composting and cocomposting of coffee husk and pulp with source-separated municipal solid waste: A breakthrough in valorization of coffee waste. International Journal of Recycling of Organic Waste in Agriculture, 8(3), 263-277. https://doi.org/10.1007/s40093-019-0256-8

Fan, Y. V., Lee, G. T., Klemeš, J. J., Ghua, L. S., Sarmidi, M. R., y Leow, G. W. (2018). Evaluation of Effective Microorganisms on home scale organic waste composting. Fournal of Environmental Management, 216, 41-48. https://doi.org/10.1016/j.jenvman.2017.04.019

FAO. (2019). The State of Food and Agriculture 2019. Moving forward on food loss and waste reduction. Rome. http:/ / www.fao.org/3/ca6030en/ca6030en.pdf

Ferronato, N., Rada, E. G., Gorritty, M. A., Gioca, L. I., Ragazzi, M., y Toretta, V. (2019). Introduction of the circular economy within developing regions: A comparative analysis of advantages and opportunities for waste valorization. Fournal of Environmental Management, 230. https://doi.org/10.1016/j.jenvman.2018.09.095 
Ferronato, N., y Torretta, V. (2019). Waste Mismanagement in Developing Countries: A Review of Global Issues. International Journal of Environmental Research and Public Health, 16(6). https://doi. org/10.3390/ijerph16061060

Kaza, S., Yao, L., Bhada-Tata, P., y Van Woerden, F. (2018). What a waste 2. 0: A global snapshot of solid waste management to 2050. The World Bank.

Morán, S. (2018). Basura: Los números rojos de Ecuador. Plan V. https://www.planv.com.ec/historias/ sociedad/basura-numeros-rojos-ecuador

Ogunlade, M. O., Bello, O. S., Agbeniyi, S. O., y Adeniyi, D. O. (2019). Microbiota Assay of Cocoa Pod Husk - Based Compost as Organic Fertilizer. International fournal of Current Microbiology and Applied Sciences, 8(06), 3182-3192. https://doi.org/10.20546/ijcmas.2019.806.380

Paritosh, K., Kushwaha, S. K., Yadav, M., Pareek, N., Ghawade, A., y Vivekanand, V. (2017). Food Waste to Energy: An Overview of Sustainable Approaches for Food Waste Management and Nutrient Recycling. BioMed Research International, 20. https://doi.org/10.1155/2017/2370927

Rahim, I., Nasruddin, A., Kuswinanti, T., Asrul, L., y Rasyid, B. (2018). Utilization of Cocoa Pod Husk Waste Composting by Tremella Sp and Pleurotus Sp as A Medium to Growth of Cocoa Seedling. IOP Conference Series: Earth and Environmental Science, 156, 012012. https://doi. org/10.1088/1755-1315/156/1/012012

Vitinaqailevu, R., y Rajashekhar Rao, B. K. (2019). The role of chemical amendments on modulating ammonia loss and quality parameters of co-composts from waste cocoa pods. International fournal of Recycling of Organic Waste in Agriculture, 8(S1), 153-160. https://doi.org/10.1007/s40093-0190285-3 
